\title{
Una aproximación del efecto en el aprendizaje de una lengua extranjera debida a la obtención de datos a través de exámenes en línea de idiomas
}

\section{An approximation to the effect on learning a foreign language due to through Big Data collection from online language exams}

\author{
Teresa Magal-Royo \\ Universitat Politécnica de Valencia, Valencia, España \\ tmagal@degi.upv.es \\ Jesús García Laborda \\ Universidad de Alcalá de Henares, Madrid, España \\ jesus.garcialaborda@uah.es
}

\begin{abstract}
Resumen
La Inteligencia Artificial orientada a la educación (AIEd) permite adecuar y/o adaptar los itinerarios del aprendizaje de un usuario mediante procesos inductivos basados en la extracción de datos obtenidos de las evidencias formativas que genera a lo largo de su vida escolar. El Big data, o datos masivos es el almacenamiento de grandes cantidades de datos que pueden ser analizados por diversos procedimientos y que permite encontrar patrones repetitivos o formulas predictivas que pueden generar un aprendizaje sobre nosotros mismos y sobre todo en la red. En el caso de los datos masivos que se generan a través de los exámenes utilizados en el aprendizaje y certificación de conocimiento de idiomas como segunda lengua a nivel nacional encontramos que podría ser útil aplicar las metodologías de procesamiento del Big Data para conocer mejor si la información generada a través de los test pueden mejorar o crear nuevas estrategias de aprendizaje o establecer criterios formales en el diseño de las pruebas, teorías de adquisición de se segunda lengua o incluso políticas educativas. La novedad de artículo se centra en establecer directrices viables para aplicar los conceptos más genéricos del Big Data en el contexto específico de los test de evaluación de idiomas como segunda lengua y donde existe a priori una gran cantidad de información a procesar a nivel educativo. El artículo muestra algunas directrices que podrían aplicarse en los mecanismos aplicados en la extracción de datos educativos del aprendizaje de idiomas a gran escala en el entorno específico de los test de evaluación de idiomas como lengua extranjera.
\end{abstract}

Palabras clave: Datos Masivos, Inteligencia Artificial orientada a la educación, Minería de datos educativos, Aprendizaje a lo largo de la vida, Evaluación en el aprendizaje de segundas lenguas.

\footnotetext{
Abstract

Artificial intelligence oriented to education (AIEd) allows the adequacy and / or adaption to the user's learning itineraries through inductive processes based on the extraction of data obtained from the formative evidences that it generates throughout its school life. Big data, or massive data, is the storage of large amounts of data that can be analyzed by various procedures and allows us to find repetitive patterns or predictive formulas that can generate learning about ourselves and especially the network. In the case of the massive data that are generated through the use of tests in the learning and certification of knowledge of languages as a foreign language at the national level, we find that it might be useful to apply Big Data's processing methodologies in order to know better if the information
} 


\begin{abstract}
Generated through the tests can improve or create new learning strategies or establish formal criteria in the design of the tests, theories of second language acquisition, or even educational policies. The novelty of the article focuses on establishing viable guidelines to apply the more generic concepts of Big Data in the specific context of the tests of language evaluation as a second language and where there is a priori a large amount of information to be processed at the educational level. The article shows some guidelines that could be applied in the mechanisms used in the extraction of educational data from large-scale language learning in the specific environment of language assessment tests as a foreign language.
\end{abstract}

Keywords: Big Data, Artificial Intelligence in Education, Educational data mining, long life learning, testing in second language learning.

\title{
1. Introducción
}

Dentro de la Inteligencia artificial orientada a la enseñanza de lenguas extranjeras, IALE, encontramos numerosas investigaciones orientadas a procesar la información que se genera durante el proceso de aprendizaje. Sin embargo, existe una amplia carencia del uso de dicha información para el aprendizaje de lenguas extranjeras. El desarrollo de entornos de aprendizaje adaptativos puede ayudar a los agentes educativos (profesores, alumnos, instituciones, etc...) a conocer, mejorar y personalizar el escenario formativo de un área concreta de conocimiento, (Bailin, 1988; Harrington, 1996; Robinson, 2005; Dodigovic, 2007; Ettlinger, Morgan-Short, Faretta-Stutenberg, \& Wong, 2016). El procesamiento de datos generados por el usuario sobre un entorno de aprendizaje puede generar formulas predictivas que ayude a mejorar las estrategias de aprendizaje educativas de carácter semiautomático (Luckin et Al., 2016) o simplemente orientar cómo mejorar su actuación especialmente en la lengua hablada (Neri, Cucchiarini, \& Strik, 2008; de Vries, Cucchiarini, Bodnar, Strik, \& van Hout, 2015).

La mayoría de las Universidades ofrecen en la actualidad, plataformas virtuales de formación en lenguas extranjeras en la red que permiten desarrollar diversas metodologías formativas en la red desde cursos virtuales masivos on-line o MOOCs, cursos semi-presenciales o repositorios de material formativo de apoyo de los cursos presenciales (Barrs, 2012; Dwaik, Jweiless, \& Shrouf, 2016; Lai, Shum, \& Tian, 2016). En todos ellos, las plataformas virtuales disponen de mecanismos de control y verificación de la comunicación entre el usuario y la plataforma mediante la generación de datos estadísticos de carácter cuantitativo y cualitativo que sirven tanto para mejora de las estrategias de aprendizaje sino para observar qué contenidos deben revisar o mejorar los estudiantes (Byrne, 2007; Godwin-Jones, 2007; Gao, Samuel, \& Asmawi, 2016).

La información gestionada por los administradores de las plataformas mediante un diseño de interfaz adecuado (García Laborda, 2009), (Magal-Royo \& García Laborda, 2017) a nivel global en una Universidad, Facultad o Escuela permite obtener valores estadísticos de carácter genérico para determinar porcentajes de éxito de una titulación, tendencias en la matriculación, número de alumnos matriculados, etc... La información gestionada por el profesor se centra se basa en la recopilación sistematizada de evidencias generadas por el alumno y que en la medida de lo posible permite evalúa

Una aproximación del efecto en el aprendizaje de una lengua extranjera debida a la obtención de datos a través de exámenes en línea de idiomas. Teresa Magal-Royo y Jesús García Laborda. Página 2 de 14 
avances y mejoras en las habilidades cognitivas sobre un tema concreto (Gilbert and Gale, 2008).

Es precisamente este hecho donde encontramos que la cantidad de información generada desde una plataforma digital para el aprendizaje de idiomas es enorme si evaluamos una institución con todos sus alumnos, las asignaturas en las que está matriculado anualmente y sobre las cuales se solicita al alumno que trabaje y gestione la información dentro de ellas, (Jewitt, 2013), (Alonso and Arranz, 2016), (Ben et al. 2009).

La evaluación de un alumno sobre una capacidad o una habilidad en una lengua extranjera pueden generarse desde una plataforma virtual y complementa la decisión final del profesor, especialmente en lo referente a la evaluación formativa (Bunch, 2011). Las herramientas de creaciones de pruebas o test on-line mediante las herramientas internas a la plataforma, permiten disponer de datos estructurados o no estructurados que generan estadísticas cuantitativas que son utilizadas por el profesor como evidencias del progreso del alumno y/o grupo de alumnos (Kumar et al. 2016).

Si nos centramos en la evaluación del aprendizaje de un idioma como lengua extranjera a lo largo de la vida formativa de un alumno en un periodo concreto como pueda ser su conocimiento de idiomas en su educación secundaria antes de la Universidad y siempre y cuando pudieran estar controladas digitalmente desde una plataforma educativa online, dispondríamos de una información valiosa sobre la evolución formativa del alumno. Es más, la obtención masiva de datos nos puede facilitar información sobre cómo se adquiere una lengua extranjera. Es decir, de acuerdo a un estudio diacrónico durante varios años con una muestra aceptable podemos observar que elementos morfosintácticos y fonológicos aparecen antes o después. Es cierto que estudios anteriores han intentado hacer dicho estudio (Wode, 1978; Ponterotto, 1987; Colson, 1992; Goldschneider, \& DeKeyser, 2005,

Si además, la información de datos generada de un alumno como microsistema se llevara a nivel regional y/o nacional como macrosistema, permitiría aplicar mecanismos de tratamiento masivo de datos de manera eficiente y adecuada para definir líneas estratégicas de formación adaptadas en el aprendizaje de idiomas, (Chen et al. 2016), (Magal-Royo \& Giménez, 2012). El tratamiento de datos masivos (en este artículo trataremos "datos masivos" o "Big Data" indistintamente) permitiría conocer la evolución de sistemas bilingües o de la competencia general de una comunidad autónoma, tema que ha sido de interés del Ministerio de Educación durante años. Es más, el poder realizar el estudio con ordenadores y usando datos masivos permitiría ampliar la batería de preguntas al automatizarse la corrección y, por tanto, ampliar la fiabilidad de la misma (Gibson \& Webb, 2015). Esto tiene un alto valor predictor para desarrollar métodos de enseñanza y políticas de mejora.

De hecho, a nivel internacional, las empresas y universidades de certificación de idiomas (British Council, Cambridge Board of Examinations, Educational Testing System, etc.) basados en test informatizados on-line manejan datos estructurados ya sean de informes contextuales (O’Loughlin, 2013) o resultados académicos (Yang \&

Una aproximación del efecto en el aprendizaje de una lengua extranjera debida a la obtención de datos a través de exámenes en línea de idiomas. Teresa Magal-Royo y Jesús García Laborda. Página 3 de 14 
Badger, 2015)que pueden ser procesados para la obtención de comportamientos educativos predictivos futuros. En la actualidad la mayoría de ellos utilizan los datos para obtener estadísticas sobre el nivel de éxito en la superación de las pruebas basadas en las competencias de evaluación de idiomas (lectura, redacción, escucha, habla). Dichos datos son visibles y consultados por investigadores e instituciones ya que son una referencia tangible de la evolución formativa de idiomas en un país o nivel educativo por ejemplo (IELTS, 2016). Sin embargo, estos datos, generalmente basados en el manejo estadístico de los grados de acierto y error de los ítems incluidos en el examen podrían, como ya hemos dicho anteriormente, aportar algo más que un análisis de errores para el desarrollo de estrategias de examen que lleven al éxito de los alumnos. Dichos datos estructurados de carácter masivo obtenidos por las Instituciones certificadoras de idiomas pueden manejarse a través de mecanismos algoritmos predictivos basados en la filosofía del Big Data y/o de mecanismos para el tratamiento de la Minería de datos educativos, EDM que permitiría obtener datos significativos sobre el aprendizaje de idiomas en un entorno de aprendizaje específico o globalizado con el uso de lo dicho anteriormente (Volk et al., 2015).

\section{Generación de datos en el aprendizaje y evaluación.}

En general, y como hemos indicado anteriormente, en el concepto general de tratamiento masivo de datos en la red y dentro de ella, las técnicas de minería de datos educacional orientada a crear sistemas de aprendizaje asistido pueden ayudar generar patrones de predicción en el aprendizaje. Para ello, el uso de información estructurada o no estructurada generada por el usuario durante un proceso concreto de aprendizaje de idiomas es fundamental para poder determinar los avances significativos en su formación. Cuando el aprendizaje se centra en un periodo de tiempo concreto de carácter reducido, la evaluación del conocimiento en el tiempo es relativamente fácil ya que el control de las evidencias puede realizarse con rapidez relativa. No obstante, cuando hablamos de un aprendizaje diacrónico a lo largo de la vida o, como mínimo, del periodo escolar como pueda ser el aprendizaje de un idioma como segunda lengua, verificamos que aunque existe un itinerario formativo concreto que empieza desde la edad temprana (en España 3 años) hasta una determinada edad cuando ya se han adquirido las competencias básicas en torno al nivel A2-B1 en el Marco Común de Referencia para las Lenguas (en torno a 12 años, final de enseñanza Primaria), el carácter cambiante, variable y adaptativo de la comunicación en un idioma, varía en función de cómo, cuándo y para qué se utiliza durante nuestra vida personal, académica o profesional. Como se ve en la figura 1, el tiempo juega un papel fundamental para ver los efectos del aprendizaje. Como se ve, mientras que la recogida de datos sincrónica solamente aporta datos parciales que se pueden tipificar para mejorar las estrategias de examen en un momento dado y en un cierto contexto (por ejemplo un determinado examen como puede ser First Certificate, Cambridge Advanced, Test of English as a Foreign Language, SAT, etc.) un estudio acumulativo de años, por ejemplo, realizado en varios colegios puede aportar una sólida estructura de datos para obtener inferencias en los métodos de enseñanza, en los factores y dificultades de aprendizaje, en la

Una aproximación del efecto en el aprendizaje de una lengua extranjera debida a la obtención de datos a través de exámenes en línea de idiomas. Teresa Magal-Royo y Jesús García Laborda.

Página 4 de 14 
evolución por la edad y en el análisis de estructuras más complejas (condicionales, voz pasiva, estilo indirecto, tec.).

Figura 1. Método y objetivos de la obtención de datos masivos.

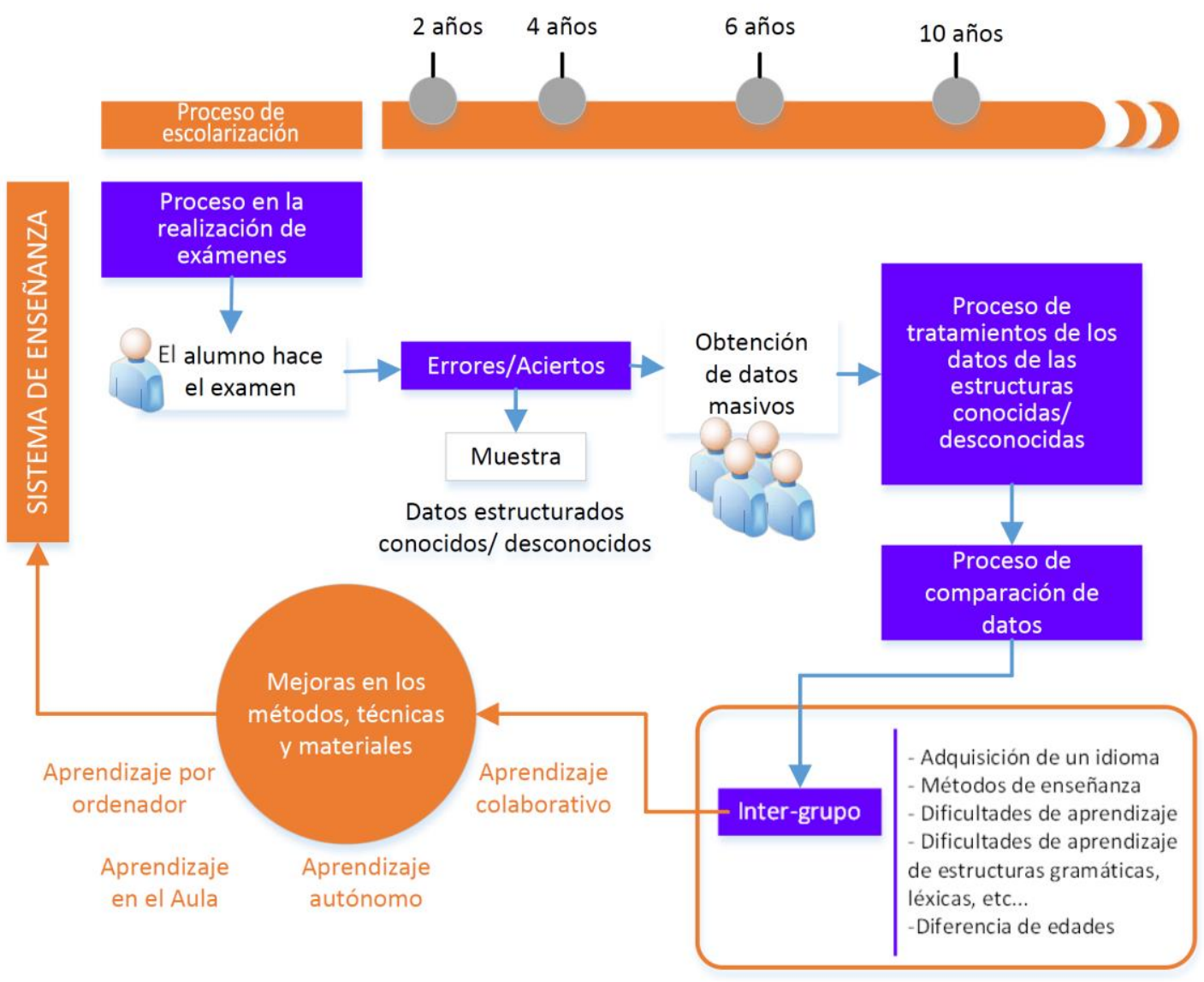

Elaboración propia, 2017

El aprendizaje a lo largo de los años escolares sobre inglés, puede llegar a generar una gran ingente cantidad de datos si éstos, por ejemplo, se generan en un entorno digital on-line que permita su registro interno tal y como en la actualidad se manejan los datos en un navegador de internet. El carácter predictivo de nuestros itinerarios de búsqueda en la red determina en ocasiones las búsquedas futuras en base a motores y algoritmos predictivos adaptados nuestras necesidades.

En el futuro, la formación cada vez más digital, está permitiendo crear algoritmos adaptativos orientados a la educación y que permiten avanzar en las teorías sobre la inteligencia artificial orientada a la Educación IAEd en base al manejo de tres tipos de datos generados por el usuario en la red y generalmente sobre una plataforma o entorno digital; los datos estructurados, los datos no estructurados y los semi-estructurados, (Guedes et Al. 2016).

Los datos estructurados (Structured Data) son la información que puede utilizarse y procesarse a través de una base de datos relacional, vienen definidos por un formato y por lo general, se guardan en tablas. Un ejemplo de datos estructurado son los

Una aproximación del efecto en el aprendizaje de una lengua extranjera debida a la obtención de datos a través de exámenes en línea de idiomas. Teresa Magal-Royo y Jesús García Laborda. Página 5 de 14 
formularios y los test on-line creados para cualquier propósito como es el caso de algunas tareas de exámenes de gramática de los exámenes certificados de idiomas. Para ello, deberemos estudiar fundamentalmente ítems de respuesta cerrada que son comunes en exámenes de idiomas largos, especialmente para inferir el conocimiento de vocabulario y gramática (Yelbay Yilmaz, 2015)

Los datos no estructurados (nonstructured Data) carecen de un formato específico para ser controlados y no disponen de información relacional o extraíble que pueda ser tratado de manera específica. Algunos ejemplos son los documentos multimedia, los correos electrónicos, documentos de texto, etc..., que puedan generarse en un entorno educativo y que son utilizados de manera interna en una clase o entre un alumno y el profesor. Este formato se puede usar para la recogida de datos en composiciones escritas libres por ordenador aunque requiere la intervención y análisis humano.

Los datos semiestructurados (Semistructured Data) que aunque son documentos de carácter no estructurado por ser creados como diferentes tipos de documentos digitales permiten crear marcadores internos conocidos como metadatos que permiten su control y seguimiento por el usuario. En este caso, encontramos ejemplos en los documentos que son cargados en plataformas educativas virtuales como evidencias para su evaluación pero que al ser introducidas en una plataforma estructurada pueden ser localizados y validados con diferentes técnicas. Este trabajo se puede hacer a través de entrevistas realizadas por ordenador que finalmente sirven para observar el uso del vocabulario en el habla a través de un análisis léxico de tokens (elementos aislados) y el uso de corpus lingüísticos.

Por otra parte, la web social puede obtenerse para obtener datos masivos especialmente de medios que les obligan a comunicarse en inglés como Facebook, WhatsApp, Tuenti y otros. Las herramientas o aplicaciones colaborativas que se implementan dentro de las plataformas educativas virtuales permiten recopilar otros tipos de datos masivos como las evidencias comunicativas de carácter formal y no formal que el estudiante genera durante su aprendizaje de la lengua extranjera. Los accesos, tipo de comunicación establecida, tiempo de respuesta, mapas de interacción, etc..., pueden ser procesados para disponer de otros datos significativos en el control del aprendizaje de un usuario en la red (Phillips et al. 2012). Los datos mediante dichas aplicaciones, pueden ser generados:

- El canal Web and los cabales del Social Media: Incluye contenidos generados para la web o creada para de las redes sociales como Facebook, Twitter, blogs, etc... y que son utilizadas también en plataformas educativas en el aprendizaje formal y no formal (Zhuravleva, de Bot, \& Hilton, 2016).

- Generados por las personas mediante herramientas de comunicación y/o de colaboración: Entre ellos encontramos las herramientas on-line y off-line como e-mails o mensajes por WhatsApp, responder a una encuesta online, acceso a navegadores, etc...(Itakura, 2004; Schenker, 2012)

- Comunicación entre máquinas o Machine-to-Machine (M2M): La gestión de datos e información mediante estas tecnologías se refiere a las que permiten conectarse entre dispositivos y también entre el usuario y uno o varios

Una aproximación del efecto en el aprendizaje de una lengua extranjera debida a la obtención de datos a través de exámenes en línea de idiomas. Teresa Magal-Royo y Jesús García Laborda.

Página 6 de 14 
dispositivos de control. Por ejemplo, el uso de sensores o medidores que capturan algún evento en particular sobre el usuario (velocidad, temperatura, presión, variables meteorológicas, variables químicas como la salinidad, etc.) los cuales transmiten a través de redes alámbricas, inalámbricas o híbridas a otras aplicaciones que traducen estos eventos en información significativa y evaluable sobre un proceso que se esté desarrollando.

Si nos centramos en los datos estructurados y semi-estructurados podemos obtener mucha información mediante las técnicas convencionales del Data mining que permiten extraer comportamientos predictivos, tendencias o patrones de uso (Hand et al., 2001). Por tanto, podemos incluso llegar a trazar la ruta que siguen los aprendices de una lengua cuando adquieren la misma e incluso observar diferencia según contextos educativos formales e informales.

Según Murphy el procesamiento de datos a gran escala se centra en la generación y selección de datos que posteriormente serán procesados mediante mecanismos de filtrado que permita sacar la información basada en patrones que deben ser presentados de manera eficaz y coherente (Murphy, 2012). En educación, la extracción de la información se utiliza en el desarrollo de procesos de máquinas de aprendizaje asistido por ordenador, (Machine learning) es un concepto derivado de la propia minería de datos y que permite el diseño de programas o algoritmos de aprendizaje que pueden aprender reglas a partir de datos, adaptar cambios instructivos sin necesidad de la mano del hombre (Shalev-Shwartz and Ben-David, 2014). Según Murphy se puede dividir en tres grandes grupos de procesos de aprendizaje automatizado:

- Aprendizaje supervisado o predictivo, en donde el ordenador aprende no sólo de los propios datos finales (inputs) sino de modelos previamente desarrollados con datos adicionales ya categorizados (outputs) para que el aprendizaje sea mucho más fiable;

- Aprendizaje no supervisado o descriptivo en el que sólo se dan los inputs al ordenador para que encuentre patrones interesantes a partir de los datos facilitados.

- Aprendizaje supervisado para realizar tareas concretas y donde las aplicaciones creadas requieren algoritmos que detecten patrones en los datos específicos.

El aprendizaje no supervisado es el que mejor se adaptaría en este caso a las pruebas de gestión de la información de los datos generados en un test de aptitud de inglés como segundo idioma ya que a diferencia del aprendizaje supervisado, utiliza procedimientos inductivos, extrayendo conocimiento sólo de los datos, como en el caso del análisis de clústers (Han et al. 2012).

Una de las aplicaciones específicas más útiles es el modelado de temas o topic modeling, que comprende la extracción de información concreta de una palabra o conjunto de palabras. Un ejemplo práctico de esta metodología aplicada a los exámenes de idiomas seria el procesamiento de los datos de los test de certificación de idiomas en cada país para obtener los temas educativos que los profesores puedan utilizar en la creación de nuevos exámenes y que, sin duda, modifica los contenidos aprendidos en el

Una aproximación del efecto en el aprendizaje de una lengua extranjera debida a la obtención de datos a través de exámenes en línea de idiomas. Teresa Magal-Royo y Jesús García Laborda. Página 7 de 14 
aula especialmente en el vocabulario (Brock-Utne, 2007). Para llevar a cabo esta tarea se podrían por ejemplo, seleccionar automáticamente palabras del corpus de textos largos y cortos de los alumnos que realicen los test on-line y que aparecen frecuentemente, lo que indicaría que tipo de preguntas o temas son los más idóneos para trabajar posteriormente en clase y cuales son demasiado repetitivos. Asimismo, en base a la tipología de datos generados y/o procesados en entornos digitales, en el futuro podremos conocer, evaluar y predecir los itinerarios formativos de un usuario a lo largo de un periodo de tiempo (Ben-Naim et al. 2009) facilitando, complementando y comprendiendo las razones y schemata que gestionan y modifican ese aprendizaje. El tratamiento de la información a través de las técnicas globales de datos masivos y en concreto mediante los procesos desarrollados en los entornos de la minería de datos educativos permite crear mapas educativos individuales y/o globalizados que se extrapolan para evidenciar el éxito o fracaso de líneas estratégicas educativas en un entorno individual y/o global (Ersanli, 2016).

\section{El procesamiento datos generada a través de los test de evaluación de aprendizaje en segundas lenguas.}

Bajo esta premisa, si nos centramos en la evaluación o certificación de segundas lenguas asistidas por ordenador de carácter internacional como el TOEFL, IELTS, etc..., donde los resultados obtenidos de las pruebas o test de conocimiento on-line son en su mayoría, datos estructurados o semi-estructurados que pueden ser procesados de forma cuantitativa y cualitativa. Este procesamiento que en la actualidad no ha sido investigado nunca desde esta nueva perspectiva permitiría predecir tendencias en el aprendizaje aplicando las técnicas de tratamiento masivo de datos.

Actualmente, las plataformas de certificación de idiomas permiten visualizan parte de la información obtenida a nivel macroescala, por ejemplo a nivel de acierto-fallo (Figura 1) cuantitativo en una competencia concreta (gramática, lectura, escritura, escucha, habla) de un país por ejemplo pero se entiende que no han trabajado suficientemente los resultados obtenidos de carácter interno que permitiría desde validar un ejercicio o tarea concreta para una competencia especifica hasta evaluar el diseño formal de las pruebas en sí mismas (García Laborda, Magal-Royo \& Bakieva,2016).

La existencia de datos masivos es evidente, teniendo en cuenta la cantidad de personas que cada año a nivel mundial accede a realizar dichas pruebas y en esencia son formalmente son las mismas a nivel global.

El control estadístico de los datos generados durante las pruebas junto con los resultados obtenidos servirían para orientar estrategias de aprendizajes avanzados incluso para evaluar cognitivamente un tipo de aprendizaje sobre otro en entornos de Inteligencia artificial orientada a la Educación, IAEd ya que ofrece un termómetro social interesante que debería potenciarse cuando se diseñen los exámenes o test de certificación de idiomas en un país, en una región concreta, en un periodo de tiempo concreto, etc...

Una aproximación del efecto en el aprendizaje de una lengua extranjera debida a la obtención de datos a través de exámenes en línea de idiomas. Teresa Magal-Royo y Jesús García Laborda. Página 8 de 14 
La reflexión que permite estas afirmaciones es que dichas instituciones con los permisos éticos correspondientes de cada usuario, podría procesar la información obtenida para ofrecer a nivel mundial tendencias y mejoras en el ámbito del aprendizaje de lenguas a nivel mundial que sirviera de marco teórico para validar nuevas estrategias y métodos.

Las formas de aprender dependen en ocasiones del entorno o escenario de aprendizaje. No es lo mismo aprender un idioma como segunda lengua en España que Turquía aunque los exámenes de certificación sean los mismos en su estructura formal. Las condiciones educativas y formativas y sociales de cada país obligan sin duda a una adaptación de los contenidos que debería analizarse anualmente en base a los datos obtenidos desde el tratamiento masivo de los exámenes realizados en años anteriores.

Por ejemplo, hay países donde los test de comprensión lectora son realizados con más facilidad y son más comprensibles debido posiblemente al uso de la misma raíz o lengua madre que si el mismo test se genera en Japón donde la comprensión lectora puede verse dificultada por el tipo de idioma de signos que utilizan en la vida real.

Por último, otro aspecto a tener en cuenta es el perfil de los usuarios que acceden a los exámenes de certificación de idiomas. Podemos encontrar estudiantes que realizan los exámenes de nivel siguiendo un itinerario formativo desde temprana edad hasta llegar a la Universidad e incluso más allá si necesitan una certificación en un ámbito profesional como sanidad o negocios, hasta persona que por necesidades de trabajo o movilidad necesitan obtener un certificado de idiomas para acceder a un trabajo o a un país concreto. Ello implica una formación a lo largo de la vida que en ocasiones es continua pero en otras es discontinua o se realiza de forma esporádica por necesidad social o profesional. La información gestionada mediante el tratamiento masivo de datos permitiría realizar adaptaciones formales de los exámenes de idiomas según criterios específicos obtenidos de resultados anteriores. También implica la adecuada formación de los profesores, administradores y responsables educativos para la mejora de la recogida de datos mediante un adecuado entrenamiento (García Laborda \& Magal Royo, 2009)

\section{Conclusiones}

El artículo ofrece una reflexión sobre las posibilidades que actualmente ofrecen los datos masivos (Big Data) de manera teórica en el ámbito de la linguística aplicada orientado al desarrollo de test on-line asistidos por ordenador en la evaluación de una segunda lengua. Aunque el planteamiento es considerado en la actualidad como pura teoría, en el futuro el uso de la minería de datos orientado a la educación permitirá la extracción de datos en otros ámbitos más genéricos o bien centrarnos en aspectos muy específicos que mejoren los aprendizajes y, aún más importante, las inferencias obtenidas de los exámenes. La posibilidad de usar y controlar la información de los datos masivos extraídos de las grandes pruebas online para la certificación de idiomas abre un gran campo de posibilidades también sobre dos aspectos interesantes relacionados con el Machine learning y el aprendizaje no supervisado capaz de generar temas (topics) o tendencias sociales a nivel mundial. El procesamiento de datos podría

Una aproximación del efecto en el aprendizaje de una lengua extranjera debida a la obtención de datos a través de exámenes en línea de idiomas. Teresa Magal-Royo y Jesús García Laborda. Página 9 de 14 
ser útil para mejorar el campo de la estadística social relacionada la educación y las estrategias de aprendizaje que cada vez son más personalizadas y a lo largo de la vida.

Presentación del artículo: 15 de Febrero de 2017

Fecha de aprobación: 15 de Marzo de 2017

Fecha de publicación: 31 de Marzo de 2017

Magal-Royo, T. y García Laborda, J. (2017). Una aproximación del efecto en el aprendizaje de una lengua extranjera debida a la obtención de datos a través de exámenes en línea de idiomas. RED. Revista de Educación a Distancia, 53. Consultado el (dd/mm/aaaa) en http://www.um.es/ead/red/

\section{Agradecimientos}

Teresa Magal Royo ha desarrollado el presente artículo gracias a la beca de estancias de investigadores Sénior en Centros extranjeros subvencionada por el Ministerio de Educación, Cultura y Deportes con el título: Evaluación de la interacción multimodal para la realización de pruebas de conocimiento de idiomas sobre dispositivos móviles inteligentes (PRX16/00171) desarrollada en el London Knowledge Lab. Departamento de Cultura, Comunicacion y Media, University College London.

\section{Bibliografía}

Alonso, V. and Arranz, O. (2016). Big Data y eLearning: A Binomial to the Future of the Knowledge Society. International Journal of Interactive Multimedia and Artificial Intelligence, 3(6), 29-33.

Bailin, A. (1988). Artificial intelligence and computer-assisted language instruction: A perspective. CALICO Journal, 5(3), 25-45.

Barrs, K. (2012). Fostering computer-mediated L2 interaction beyond the classroom. Language Learning y Technology, 16(1), 10-25.

Ben-Naim, D., Bain, M. and Marcus N. (2009). A User-Driven and Data-Driven Approach for Supporting Teachers in Reflection and Adaptation of Adaptive Tutorials. Proceedings of Educational Data Mining 2009, 2nd International Conference on Educational Data, in Cordoba. Mining, Barnes, T., Desmarais, M., Romero, C., y Ventura, S. (Eds.), Córdoba, pp. 21-30, 2009.

Brock-Utne, B. (2007). Learning through a familiar language versus learning through a foreign language--A look into some secondary school classrooms in Tanzania. International Journal of Educational Development, 27(5), 487-498.

Bunch, M. B. (2011). Testing english language learners under no child left behind. Language Testing, 28(3), 323-341.

Una aproximación del efecto en el aprendizaje de una lengua extranjera debida a la obtención de datos a través de exámenes en línea de idiomas. Teresa Magal-Royo y Jesús García Laborda. Página 10 de 14 
Byrne, T. (2007). Marrying two existing software packages into an efficient online tutoring tool. Computer Assisted Language Learning, 20(5), 459-469.

Chen M., Zhang Q., Huang Ch. and Chen T. (2016) The positioning and construction of education ecosystem base on Big Data. Proceedings in 2nd International Conference on Information Management (ICIM). London, England.

Colson, P. J. (1992). The acquisition of dutch as a foreign language: Natural order, interference and monitoring. Rassegna Italiana Di Linguistica Applicata, 25(3), 115-132.

de Vries, B. P., Cucchiarini, C., Bodnar, S., Strik, H., y Van Hout, R. (2015). Spoken grammar practice and feedback in an ASR-based CALL system. Computer Assisted Language Learning, 28(6), 550-576.

Dodigovic, M. (2007). Artificial intelligence and second language learning: An efficient approach to error remediation. Language Awareness, 16(2), 99-113.

Dwaik, R., Jweiless, A., y Shrouf, S. (2016). Using blended learning to enhance student learning in american literature courses. Turkish Online Journal of Educational Technology - TOJET, 15(2), 126-137.

Ersanli, C. Y. (2016). Investigation of pre-service english language teachers' cognitive structures about some key concepts in approaches and methods in language teaching course through word association test. Journal of Language and Linguistic Studies, 12(1), 124-136.

Ettlinger, M., Morgan-Short, K., Faretta-Stutenberg, M., y Wong, P. C. (2016). The relationship between artificial and second language learning. Cognitive Science, 40(4), 822-847.

Gao, X., Samuel, M., y Asmawi, A. (2016). Online features of qzone weblog for critical peer feedback to facilitate business english writing. Journal of Educational Technology Systems, 45(2), 285-301.

García Laborda, J. (2009). Interface architecture for testing in foreign language education. Procedia Social and Behavioral Sciences 7, 2754-2757.

García Laborda, J., Magal Royo,T y Bakieva, M. (2016). Looking towards the Future of Language Assessment: Usability of Tablet PCs in Language Testing. Journal of Universal Computer Science 22(1), 114-123

García Laborda, J., y Magal Royo, T. (2009). Training senior teachers in compulsory computer based language tests. Procedia Social and Behavioral Sciences 1, 141144.

Gibson, D. C., y Webb, M. E. (2015). Data science in educational assessment. Education and Information Technologies, 20(4), 697-713.

Una aproximación del efecto en el aprendizaje de una lengua extranjera debida a la obtención de datos a través de exámenes en línea de idiomas. Teresa Magal-Royo y Jesús García Laborda. Página 11 de 14 
Gilbert L., y Gale V. (2008). Principles of e-learning systems engineering. Chandos publishing. Information professional series. Oxford.

Godwin-Jones, R. (2007). Tools and trends in self-paced language instruction. Language Learning \& Technology, 11(2), 10-17.

Goldschneider, J. M., y DeKeyser, R. M. (2005). Explaining the "natural order of L2 morpheme acquisition" in English: A meta-analysis of multiple determinants. Language Learning, 55, 27-77.

Griffin, P., Care, E., Vista, A. and Scoular, C. (2014). Using innovative measurement tools based on Big Data analytics to assess collaborative problem-solving skills and improve teaching strategies in essential skills for 21 st century education. EDULEARN14: 6th International Conference on Education and new learning technologies. EDULEARN Proceedings. Edited Chova L.G. and Martinez, A.L.; Torres, I.C. pp. 6364-637.

Guedes, Á.L.V., Azevedo, R.G.d.A. and Barbosa, S.D.J (2016). Extending multimedia languages to support multimodal user interactions. Multimedia Tools and Applications 75(1), pp 1-30.

Han J., Kamber M. and Pei J. (2012). Datamining. Concepts and techniques. Morgan Kaufmann Publishers, San Francisco.

Hand D. J., Mannila, H. and Smyth P. (2001). Principles of data mining. MIT Press Books, Cambridge.

Harrington, M. (1996). Intelligent computer-assisted language learning. On-Call, 10(3), 2-9.

IELTS (2016). IELTS. Test statistics. Recuperado de https://www.ielts.org/teachingand-research/test-statistics

Itakura, H. (2004). Changing cultural stereotypes through E-mail assisted foreign language learning. System: An International Journal of Educational Technology and Applied Linguistics, 32(1), 37-51.

Jewitt C. (2013). Learning and communication in digital multimodal landscapes. London, Institute of Education Press.

Kumar Udupi P., Sharma N. and Jha S.K. (2016) Educational data mining and big data framework for e-learning environment. 5th International Conference on Reliability, Infocom Technologies and Optimization (Trends and Future Directions) (ICRITO) pp. $258-261$.

Lai, C., Shum, M., y Tian, Y. (2016). Enhancing learners' self-directed use of technology for language learning: The effectiveness of an online training platform. Computer Assisted Language Learning, 29(1), 40-60.

Luckin, R. Holmes, W. Griffiths, M., y Forcier, L.B. (2016). Intelligence Unleashed. An Argument for Artificial Intelligence in Education. London: Pearson.

Una aproximación del efecto en el aprendizaje de una lengua extranjera debida a la obtención de datos a través de exámenes en línea de idiomas. Teresa Magal-Royo y Jesús García Laborda. Página 12 de 14 
Magal-Royo T. y Garcia Laborda J. (2017) Multimodal Interactivity in Foreign Language Testing. En Multimodal Interaction with W3C Standard. Toward Natural User Interfaces to Everything, Capítulor 19. Dahl, Deborah (Ed.). Springer International Publishing. ISBN: 978-3-319-42816-1.

Magal-Royo, T., Gimenez López. J.L. (2012). Multimodal Interactivity in the Foreign Language Section of the Spanish University Admission Examination. Revista de Educacion. $\mathrm{n}^{\mathrm{o}}$ 357, pp.163-176.

Murphy, K. (2012). Machine learning. A probabilistic perspective. Cambridge/London: The MIT Press.

Neri, A., Cucchiarini, C., y Strik, H. (2008). The effectiveness of computer-based speech corrective feedback for improving segmental quality in L2 Dutch. $\operatorname{ReCALL}, 20(2), 225-243$.

O'Loughlin, K. (2013). Developing the assessment literacy of university proficiency test users. Language Testing, 30(3), 363-380.

Phillips, R., Maor, D., Preston, G. y Cumming-Potvin, W. (2012). Exploring Learning Analytics as Indicators of Study Behaviour. In T. Amiel y B. Wilson (Eds.), Proceedings of EdMedia: World Conference on Educational Media and Technology 2012 pp. 2861-2867.

Ponterotto, D. (1987). Morpheme acquisition order in italian learners of english. Rassegna Italiana Di Linguistica Applicata, 19(3), 105-119.

Robinson, P. (2005). Cognitive abilities, chunk-strength, and frequency effects in implicit artificial grammar and incidental L2 learning: Replications of reber, walkenfeld, and hernstadt (1991) and knowlton and squire (1996) and their relevance for SLA. Studies in Second Language Acquisition, 27(2), 235-268.

Schenker, T. (2012). Intercultural competence and cultural learning through telecollaboration. CALICO Journal, 29(3), 449-470.

Shalev-Shwartz S. and Ben-David S. (2014). Understanding Machine Learning: From Theory to Algorithms. Cambridge University Press

Volk, H., Kellner, K., Wohlhart, D, (2015) Learning Analytics for English Language Teaching. Journal of Universal Computer Science 21 (1) pp. 156-174.

Wode, H., Bahns, J., Bedey, H y Frank, W. (1978). Developmental sequence: an alternative approach to morpheme order. Language Learning 28, 175-185.

Yang, Y., y Badger, R. (2015). How IELTS preparation courses support students: IELTS and academic socialisation. Journal of further and Higher Education, $39(4), 438-465$.

Una aproximación del efecto en el aprendizaje de una lengua extranjera debida a la obtención de datos a través de exámenes en línea de idiomas. Teresa Magal-Royo y Jesús García Laborda. Página 13 de 14 
Yelbay Yilmaz, Y. (2015). Learning vocabulary in a foreign language: A computer software based model attempt. Journal of Language and Linguistic Studies, 11(2), 23-38.

Zhuravleva, A., de Bot, K., y Hilton, N. H. (2016). Using social media to measure language use. Journal of Multilingual and Multicultural Development, 37(6), 601-614. 